Signal \& Image Processing : An International Journal (SIPIJ) Vol.5, No.3, June 2014

\title{
SQUEEZING OF COLOR IMAGE USING DUAL TREE COMPLEX WAVELET TRANSFORM BASED PRELIMINARY PLAN
}

\author{
Nitin Sharma, Anupam Agarwal and Pawan Kumar Khyalia \\ Dept.of ECE, Jagannath University, Jaipur, Rajasthan, India
}

\begin{abstract}
In this paper, we scrutinize the role of dual tree complex wavelet transform. This Dual tree complex wavelet transform (DT-CWT) is slightly short of shift invariant and directionally selective in two or rise up dimensions.The nature of multidimensional DT-CWT is non separable \& dependent on the computationally expeditious, separable filter bank(SFB).This paper explains the designing of complex wavelet transform with directional properties and use of this designed form in squeezing of image. If we take the DT-CWT transform, then many of wavelet coefficients are approximately zero and this shows the intra sub-bands dependency. We further access the performance of SPIHT coding preliminary plan for coding of those coefficients. At last, In the results of proposed preliminary plan gives higher rate of squeezing and lower mean square error(MSE) compared to plan of DWT. Dual tree complex wavelet transform-SPIHT preliminary plan outperform DWT based preliminary plan at lower bit rates.
\end{abstract}

\section{KEYWORDS}

Image squeezing, Complex wavelet transform, Texture of image, Dual tree, SPIHT

\section{INTRODUCTION}

The discrete wavelet transform are used for squeezing the image since 1990.In the 2D-DWT squeezing technique, two one dimension are used for vertical \& horizontal direction respectively [1].The image have two types of singularities in which the 2-D discrete wavelet transform (Traditional 2-D DWT) are able to capture point singularities with more effectiveness but at the time of capturing line singularities it becomes failed. It got failed because alignment of horizontal or vertical direction of image and edges \& contour in images are not perfect. This imperfectness can be solving by using a new transform by filtering the image in both direction. If the alignment of edges \& contour are not perfect with horizontally \& vertically then the energy of image is spread across the sub band which is the property of DWT.To solve the problem of energy spreading in sub bands , the directional transform is required.

There are two categories are defined for adaptive transform which are: - First category is used for analysis the image along the set of direction which is predetermined [8].Second category is used for analysis the direction itself to the orientation feature of image [5],[11][12]. On the other hand, two types of adaptive wavelet transform \& lifting structured based transform are proposed because both are suitable for filtering the direction to the orientation of edges \& texture[5],[11].To minimize the prediction error, use different types of direction selection method which is given in [12],[15],[16]. 
Signal \& Image Processing : An International Journal (SIPIJ) Vol.5, No.3, June 2014

In this paper, we access the result of squeezing preliminary plan based dual tree CWT \& DWT with the coding plan of SPIHT (bior 4.4 filter coefficients).After analysis, the result of squeezing of preliminary plan, the proposed preliminary plan outperform than DWT plan at lower bit rate.

There are two scientist named reeves \& Kingsbury have already discussed the DT-DWT which gives the result with higher PSNR at same bit per pixel [19].Coding of image with DT-DWT is also reported [17]

\section{2-D DUAL TREE CWT}

We know that the DWT is very useful plan for signal processing but the performance of DWT is small in range because of unable to select higher dimension, aliasing problem at the time of signal in down sampled, the energy division at the time of high frequency band. The condition of aliasing can be removed by complex wavelet transform which provides the quadrature wavelets [11].

\subsection{D Dual Tree Wavelet:-}

The dual tree CWT preliminary plan gives the exact reconstruction of input signal but this plan use the analytical filter at the place of real filter for removing the deficiency of DWT plan. The dual tree CWT preliminary plan uses two DWT trees of real filter coefficient. The two wavelets wreal $(t)$ and $\psi$ imag $(t)$ are orthogonal to each other which composed as analytical function $\psi c(t)$.

On the other hand, there are two scaling function $\phi r(t)$ and $\phi i(t)$.This analytic function \& scaling function relationship is written:

$$
\begin{gathered}
\psi(t)=\sqrt{2} \sum_{n} h 1(n) \phi(2 t-n) \\
\phi(t)=\sqrt{2} \sum_{n} h 0(n) \phi(2 t-n)
\end{gathered}
$$

Scaling filter $-\mathrm{h}(0) \& \mathrm{~g}(0)$ are HT pair

Follow half sample delay condition is given by:-

$$
\mathrm{g} 0(\mathrm{n})=\mathrm{hO}(\mathrm{n}-0.5)
$$

\section{In frequency domain,}

Magnitude is given by:

$$
\left|G 0\left(e^{j w}\right)\right|=\left|I I 0\left(e^{j w}\right)\right|
$$

Phase condition is given by:

$$
\angle G 0\left(e^{j w}\right)=\angle H O\left(e^{j w}\right)
$$

In this preliminary plan, the standard DWT operates in parallel in which first one standard DWT is called real tree(tree-a) and second one standard DWT is called imaginary tree(tree-b).The conjugate filter for standard DWT is represented by ha $\&$ gb respectively.

\section{For tree a:-}

$$
h \mathrm{~h}=\left\{\mathrm{h}_{1} \mathrm{~h}, \mathrm{~h} 1\right\}
$$


Signal \& Image Processing : An International Journal (SIPIJ) Vol.5, No.3, June 2014

\section{For tree b:-}

$$
g b=\{g 0, g 1\}
$$

For synthesis, the filter pair are used.

$\{\widetilde{h 0}, \widetilde{h 1}\}$ and $\{\widetilde{g 0}, \widetilde{g 1}\}$

The dual tree CWT can be represented by matrix. When the two real DWT are represented by square matrix (Fh \& Fg):-

$$
F=\left(\begin{array}{l}
F h \\
F g
\end{array}\right)
$$

\section{For synthesis filter:}

$$
F^{-1}=\frac{1}{2}\left[F h^{-1} F^{-1}\right]
$$

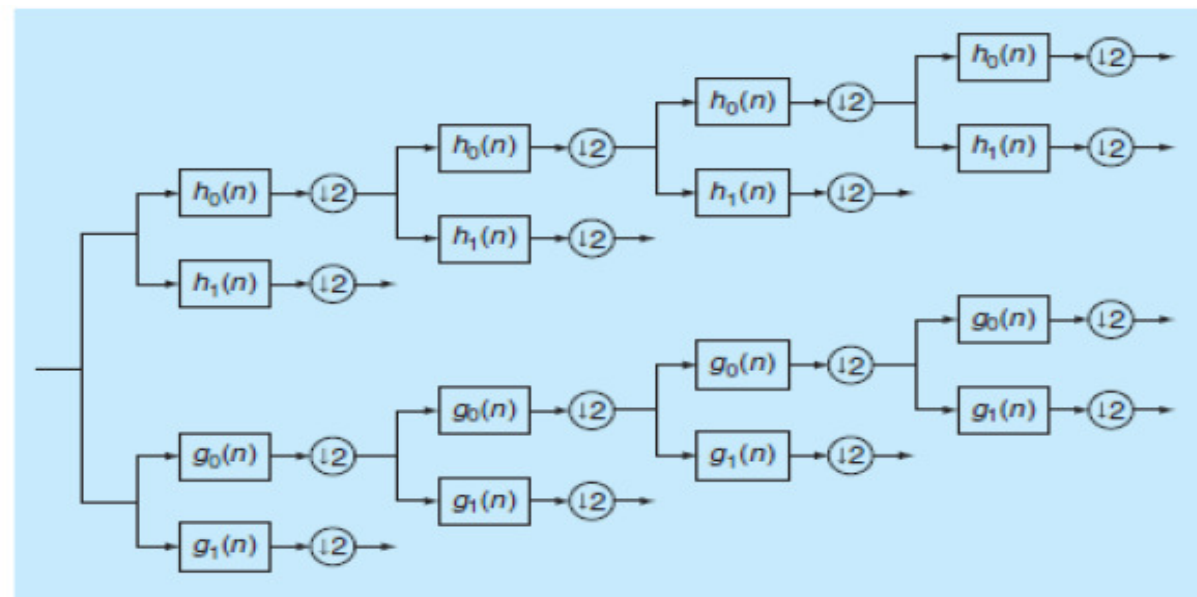

Figure-A: - Filter Bank Analysis for 1-D Dual Tree CWT

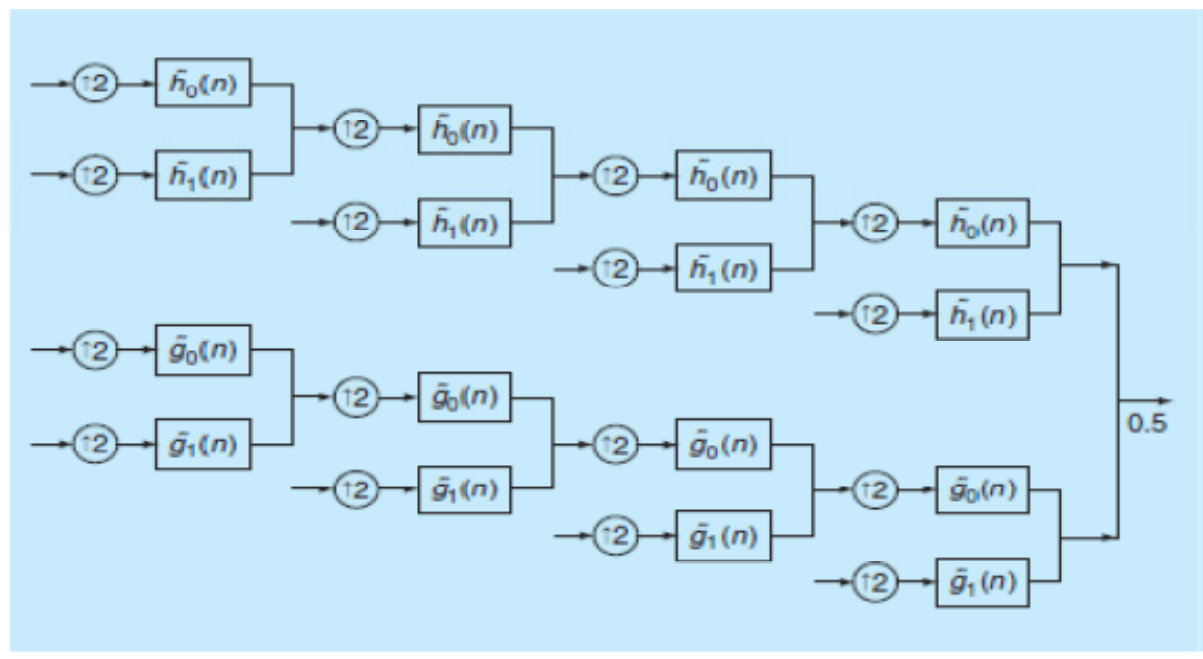

Figure-B: - Filter Bank Synthesis For 1-D Dual Tree CWT 
Signal \& Image Processing : An International Journal (SIPIJ) Vol.5, No.3, June 2014

\subsection{D Dual Tree Wavelet}

The execution of Dual tree CWT is done without evasion. At the beginning, by using two set of filter, the input image is decomposed and filtered the image horizontally and vertically. This set of filter should satisfy the PR condition and every set contain both low pass filter and high pass filter.

On the other hand, when we joint analytical filter bank and synthesis filter bank then overall transform is similar to analytical.

\section{Analysis Filter Bank Synthesis FB}

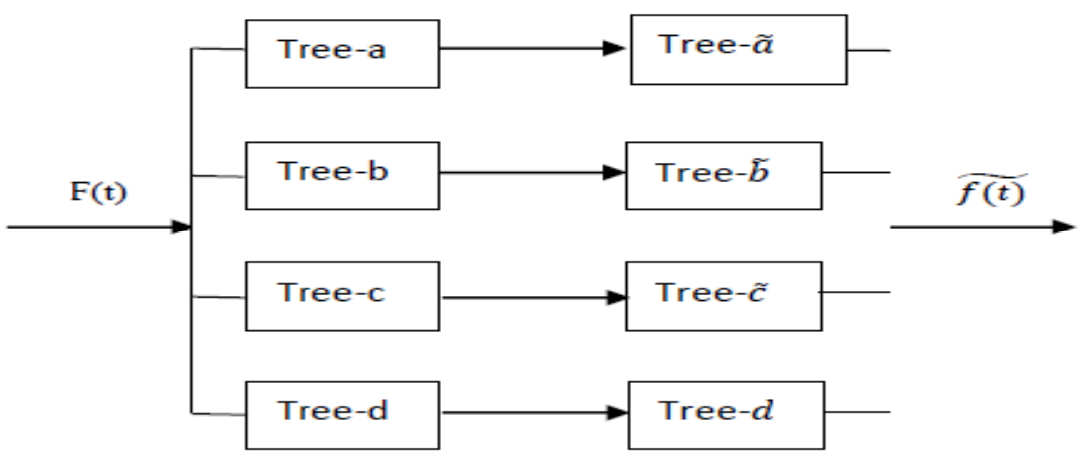

Figure -C:- Filter Bank Sructure For 2-D Dual Tree CWT

At each level of decomposition,two low pass sub band and six high pass sub bands (HLa, LHa, $\mathrm{HHa}, \mathrm{HLa}, \mathrm{LHb} \& \mathrm{HHb}$ ) are generated.

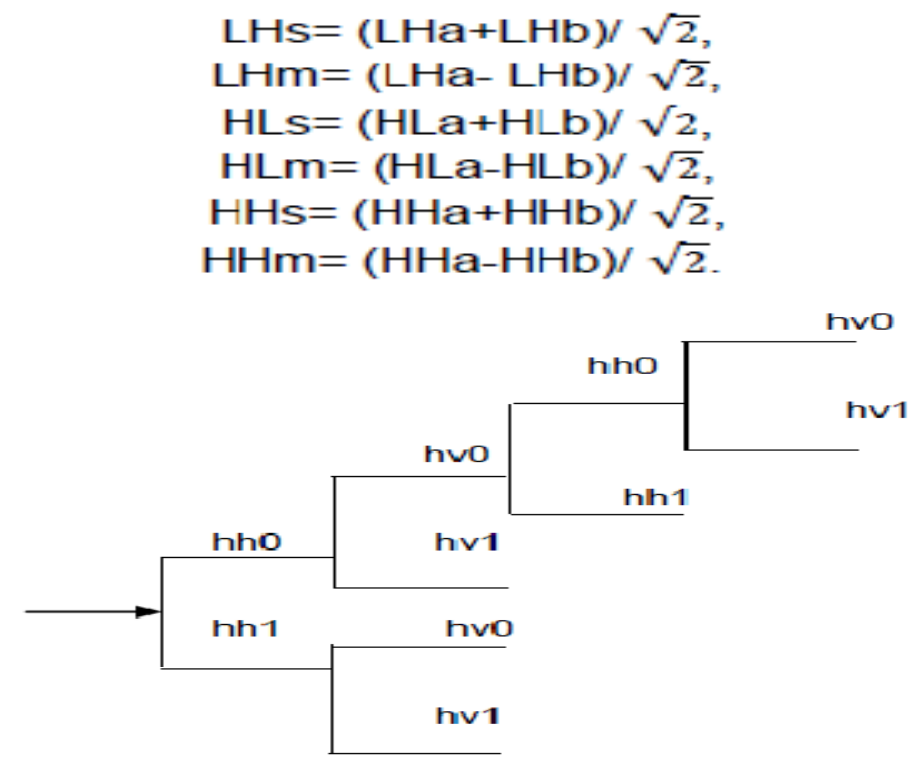

Figure-D:- Structure of Filter Bank of Tree-a

The applied filter on horizontal and vertical dimensions can be written as:-

$$
(h h+j g h)(h v+j g v)=(h h \cdot h v-g h . g v)+\mathrm{j}(h h . g v+g h . h v)
$$


Signal \& Image Processing : An International Journal (SIPIJ) Vol.5, No.3, June 2014

\section{SQUEEZING ALgORITHM \& CODING OF IMAGE}

There are two types of algorithm is necessary for squeezing \& de squeezing of image.

\section{A) Algorithm for squeezing:-}

There are five steps which covers the algorithm for squeezing.

Step 1) In the first step, the image is converted in the form of digital and read by using the MATLAB software.

Step 2) In second step, the format of image in RGB is converted into the format of $\mathrm{YCbCr}$.

Step 3) After that the component of image (y, $\mathrm{Cb}, \mathrm{Cr})$ is separated.

Step 4) Use Dual tree CWT preliminary plan for decomposition of each component.

Step 5) At last, by using SPIHT coder, the coefficient of each component is coded

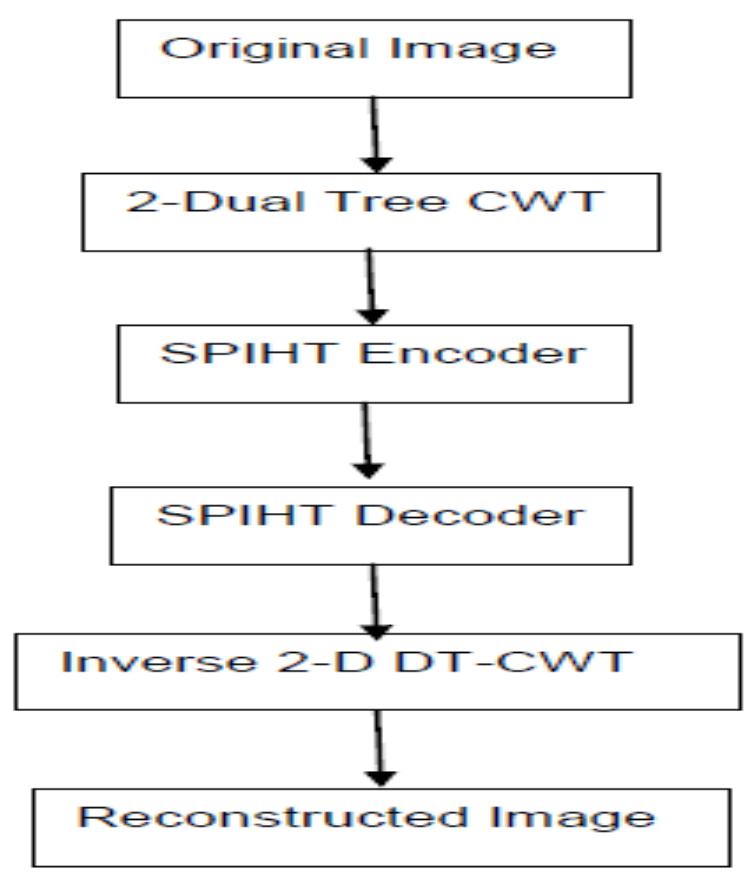

Figure-E: - Algorithm for Image Squeezing

\section{B) Algorithm for De Squeezing:-}

There are five steps which covers the algorithm of de squeezing.

Step 1) Firstly, coded image is read.

Step 2) In this step, SPIHT encoder is used to decode the coded image.

Step 3) After that, this decoded image is passed through the inverse DT-CWT

Step 4) The format of $\mathrm{YCbCr}$ is converted into RGB format.

Step 5) At last, measure the mean square error (MSE) and peak signal to noise ratio (PSNR). 
Signal \& Image Processing : An International Journal (SIPIJ) Vol.5, No.3, June 2014

\section{Performance With Coding \& Experimental Results}

The results of coded images are compared between JPEG 2000 Squeezing arrangement \& 2D DT-CWT plan that's why the transform having the ability of selection of direction. It outperform the DWT based squeezing plan here SPIHT coding plan is utilized to organize the squeezed bit stream in squeezing preliminary plan.

Squeezing ratio=Set as the input of squeezing system

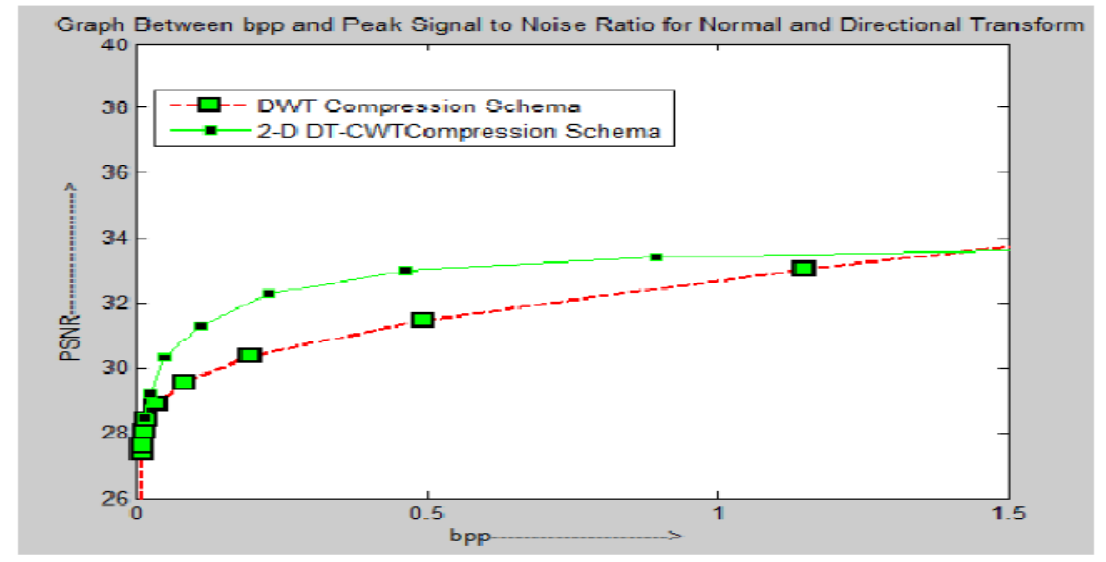

Figure-F:- Analysis of Performance Using JPEG2000 and Proposed Squeezing plan

Thus, for performance checking in image squeezing, the comparison between the JPEG and proposed preliminary plan is shown in table. The unit of performance of image is decimals. By this figure F, we can say that the proposed preliminary plan is better than the old DWT-SPIHT squeezing plan

\begin{tabular}{|l|c|c|c|c|}
\hline Image & Size & bpp & $\begin{array}{c}\text { JPEG } \\
2000\end{array}$ & $\begin{array}{c}\text { Proposed } \\
\text { schema }\end{array}$ \\
\hline \multirow{3}{*}{ Image } & $256^{*} 256$ & 0.1 & 27.22 & 27.32 \\
\cline { 4 - 6 } & & 0.25 & 30.43 & 30.49 \\
\cline { 3 - 6 } & & 0.5 & 31.04 & 32.25 \\
\cline { 3 - 5 } & & 1 & 31.79 & 33.24 \\
\hline
\end{tabular}

\begin{tabular}{|l|c|c|c|c|}
\hline Lena & $256^{\star} 256$ & 0.1 & 28.34 & 28.94 \\
\cline { 3 - 5 } & & 0.25 & 33.74 & 34.30 \\
\cline { 3 - 5 } & & 0.5 & 3701 & 3740 \\
\cline { 3 - 5 } & & 1 & 40.6 & 40.77 \\
\hline Baboon & $256^{\star 2} 256$ & 0.1 & 21.34 & 21.46 \\
\cline { 3 - 5 } & & 0.25 & 23.06 & 23.29 \\
\cline { 3 - 5 } & & 0.5 & 25.48 & 25.78 \\
\cline { 3 - 5 } & & 1 & 29.01 & 29.47 \\
\hline
\end{tabular}

Table-1:- Comparison in Performance for Different Image Squeezed by JPEG2000 \& Proposed Preliminary Plan 
Signal \& Image Processing : An International Journal (SIPIJ) Vol.5, No.3, June 2014

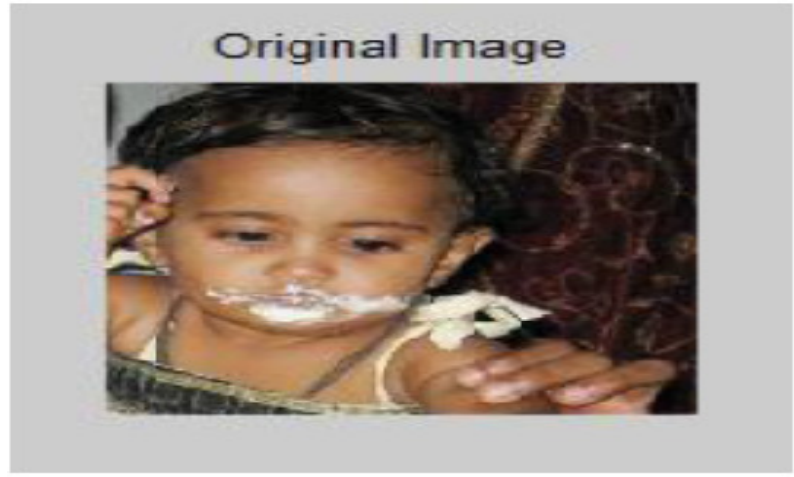

Figure-G:- Original Image

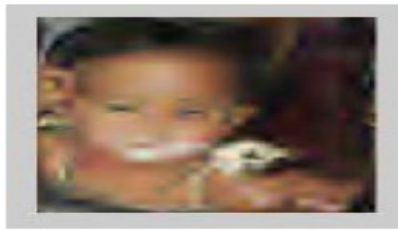

(a)

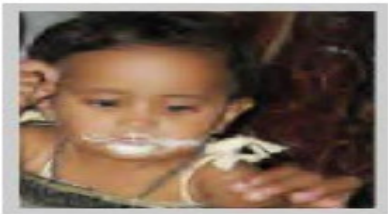

(c)

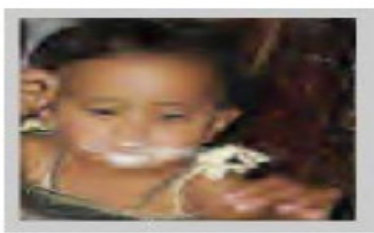

(b)

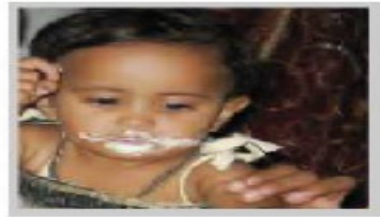

(d)

Figure-H: - Reconstructed image at (a) 0.1bpp (b) 0.25bpp (c) 0.5bpp (d) $1 \mathrm{bpp}$

\section{CONCLUSION}

In this paper, the 2-D Dual tree CWT method is presented. The image squeezing result of this proposed transform is better than JPEG2000 plan at low bit rate. This conclusion results performs on MSE \& PSNR.

\section{FUTURE WORK}

For future work, this arrangement can be used for video coding which is based on wavelet at low computational complexity

\section{ACKNOWLEDGEMENT}

A work owes its success from commencement to completion; some people have contributed solely in various stages of our paper. In this phrase, we would like to express our sincere gratitude towards Mr. Nitesh Kumar Dixit (Head of Department, Bhartiya Institute of Engg. \& Technology, Sikar), Mr.Anupam Agarwal (Assistant Professor, Jagannath University, Jaipur), Mr.Gurudutt Sharma (Senior Lecturer, Bhartiya Institute of Engg. \& Technology, Sikar) to motivate us for developing the paper. Last but not least we solely want to thank from the core of our heart to our parents and ask their heavenly blessings. We also want to thank our siblings for their moral support and constant appreciation during writing the paper. 
Signal \& Image Processing : An International Journal (SIPIJ) Vol.5, No.3, June 2014

\section{REFERENCES}

[1] Image Compression Fundamentals, Standards, and Practice. Norwell, MA: Kluwer, 2001.

[2] B. Wang, et al., "An investigation of 3D dual-tree wavelet transform for video coding", in Proc. Int.Conf. Image Processing, Singapore, Oct. 2004.

[3] Schumpert, J. and Jenkins, "A two-component image coding scheme based on two-dimensional interpolation and the discrete cosine transform ", IEEE International Conference on ICASSP, vol.-8, pp.1232-1236, April 1983.

[4] Lewis, A.S. and Knowles, G., "Image compression using the 2-D wavelet transform", IEEE Transactions on Image Processing, vol.-1, pp. 244 - 250, Apr 1992

[5] R. L. Claypoole, G. M. Davis, W. Sweldens, and R. G. Baraniuk, "Nonlinear wavelet transforms for image coding via lifting,” IEEE Trans. Image Process., vol. 12, no. 12, pp. 1449-1459, Dec. 2003

[6] Hyung Jun Kim, "Image compression using bi-orthogonal wavelet transforms with multiplier less 2D filter mask operation", Proceedings of the 1997 International Conference on Image Processing (ICIP '97), vol.-1, pp. 648-652, July 1997.

[7] Fukutomi, T. Tahara, O. Okamoto and Minami, "Encoding of still pictures by a wavelet transform and singular value decomposition", IEEE canadian Conference on Electrical and Computer Engineering, vol.- 1, pp. 16 - 23, May 1998.

[8] D. Taubman and A. Zakhor, "Orientation adaptive subband coding of images," IEEE Trans. Image Process., vol.3, no. 4, pp. 421-437, Jul. 1994.

[9] J. M. Shapiro, "Embedded Image Coding Using Zero trees of Wavelet Coefficients," IEEE Trans. on Signal Processing, vol.- 41, no.-12, pp. 3445-3462, December 1993.

[10] A. Said and W. A. Pearlman, "A New Fast and Efficient Image Codec Based on Set Partitioning in Hierarchical Trees," IEEE Trans. on Circuits and Systems for Video Technology, vol.- 6, no.-3, pp. 243-250, June 1996.

[11] Weisheng Dong, Guangming Shi, Member, IEEE, and JizhengXu, Member, IEEE," Adaptive Nonseparable Interpolation for Image Compression With Directional Wavelet Transform" in IEEE SIGNAL PROCESSING LETTERS, VOL. 15, 2008.

[12] O. N. Gerek and A. E. Cetin, "A 2-D orientation-adaptive prediction filter in lifting structures for image coding,” IEEE Trans. Image Process., vol. 15, no. 1, pp. 106-111, Jan. 2006.

[13] W. Ding, F. Wu, X. Wu, S. Li, and H. Li, "Adaptive directional lifting-based wavelet transform for image coding,” IEEE Trans. Image Process., vol. 16, no. 2, pp. 416-427, Feb.2007.

[14] S. Mallat, "A theory for multiresolution signal decomposition", IEEE Transactions on Pattern Analysis and Machine Intelligence, vol.-11, issue-7, pp. 674-683, July 1990.

[15] Weisheng Dong, Guangming Shi, Member, IEEE, and JizhengXu, Member, IEEE," Adaptive Nonseparable Interpolation for Image Compression With Directional Wavelet Transform" in IEEE SIGNAL PROCESSING LETTERS, VOL. 15, 2008.

[16] C.-L. Chang, A. Maleki, and B. Girod, "Adaptive wavelet transform for image compression via directional quincunx lifting," in Proc. IEEE Workshop Multimedia Signal Processing, Shanghai,China,Oct. 2004.

[17] T. H. Reeves and N. G. Kingsbury, "Overcomplete image coding using iterative projection- based noise shaping”, in Proc. Int. Conf. Image Processing, Rochester, NY, Sept 2002.

[18] Jingyu Yang, Yao Wang, Wenli Xu and Qionghai Dai," Image Coding Using Dual-Tree Discrete Wavelet Transform”, IEEE Transactions on Image Processing, vol.-17 pp. 1555-1569, Sept. 2008.

[19] N. G. Kingsbury and T. H. Reeves, "Redundant representation with complex wavelets: how to achieve sparsity", in Proc. Int. Conf. Image Processing, Barcelona, Sept. 2003.

[20] N. G. Kingsbury and J. F. A. Magarey, "Wavelet Transforms in Image Processing", Proceeding of First European Conference on Signal Analysis and Prediction, pp. 23-24, June 1997.

[21] Schumpert, J. and Jenkins, "A two-component image coding scheme based on two-dimensional interpolation and the discrete cosine transform ", IEEE International Conference on ICASSP, vol.-8, pp. 1232-1236, April 1983.

[22] Haque, "A two-dimensional fast cosine transform", IEEE Transactions on ICASSP, vol. - 33, Issue:6 on pp. 1532 - 1539, Dec 1985.

[23] Olivier Rioul and Martin Vetterli, "Wavelets and Signal Processing", IEEE Trans. on Signal Processing, vol.-8, Issue 4, pp. 14 -38, October 1991. 
Signal \& Image Processing : An International Journal (SIPIJ) Vol.5, No.3, June 2014

\section{AUTHORS}

NITIN SHARMA is a Lecturer in Deptt. of Electronics \& Communication Engg.He received B-Tech (ECE) degree from Sobhasaria Group of Institution, Sikar with hons. Marks in 2012 and M-TECH (Digital Communication) degree from Jagannath University, jaipur in 2014.He has two years experience in teaching. He has published research paper in international journal. Some of his work interest includes audio, speech, image, biological signals.

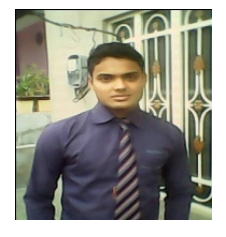

ANUPAM AGARWAL is Assistant Professor in Department of ECE in Jagannath University, Jaipur. He has received B.E (ECE) degree from BIET, Jhansi (U.P) in 2009 \& M-Tech (Power Electronics) degree from NIT, Khrukshetra in 2011. He has got two years \& six month of experience in research \& teaching. He has published research papers in national \& international journals and attendant a no. of conferences. His current areas of interest include Image and Video Processing, Power Electronics, Embedded Systems.

PAWAN KUMAR KHYALIA has done B-TECH from Sobhasaria Group of Institution.M-tech perusing from jagannath university, Jaipur
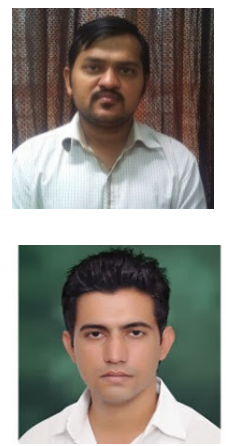